Macedonian Pharmaceutical Bulletin, 66 (Suppl 1) 123 - 124 (2020)

Online ISSN 1857 - 8969

UDC: 606:577.216]:613.25

DOI: 10.33320/maced.pharm.bull.2020.66.03.061

Short communication

\title{
Membrane interactions and cellular uptake of an amphipathic cell-penetrating peptide as a delivery system for miRNA
}

\author{
Ivana Ruseska $^{1}$, Anna-Laurence Schachner-Nedherer ${ }^{2}$, Andreas Zimmer ${ }^{1}$ \\ ${ }^{1}$ Institute of Pharmaceutical Sciences, Department of Pharmaceutical Technology and Biopharmacy, \\ University of Graz, Universitätsplatz 1, 8010 Graz, Austria \\ ${ }^{2}$ Division of Biophysics, Gottfried Schatz Research Center for Cell Signaling, Metabolism and Aging, \\ Medical University of Graz, Neue Stiftingtalstraße 6, 8010 Graz, Austria
}

\section{Introduction}

Cell-penetrating peptides constitute a promising strategy for the intracellular delivery of therapeutic molecules, such as nucleic acids. The non-covalent approach based on the amphipathic N-TER peptide has shown to be successful in the intracellular delivery of miRNA-27a by inducing its antiadipogenic effect in 3T3-L1 cells. However, information regarding the events taking place at the cellular interface is lacking. Furthermore, information on the uptake process often comes as confusing and contradictive. The main focus of our study is to examine how the peptide interacts with the cell membrane, is this interaction fatal for the cells, or is it just the first step towards the peptide's internalization into the cells?

\section{Materials and methods}

\section{Materials}

The N-TER peptide is part of the "N-TER Nanoparticle siRNA Transfection System" from Sigma Aldrich (Vienna, Austria). For the complexes, three different nucleic acids from Dharmacon were used (GE Healthcare, Vienna, Austria): double stranded miRNA mimic (miRNA-27a), miRNA mimic used as a non-targeting control (NTC) and miRNA mimic transfection control with Dy547 (Fluo-NTC). For in vitro experiments, mouse embryonic fibroblast-derived 3T3-L1 preadipocytes were used.

\section{Sample preparation}

To obtain standard N-TER peptide-nucleic acid complexes, aqueous solutions of the N-TER peptide and the respective nucleic acid (miRNA-27a, NTC or Fluo-NTC) were mixed in well-defined ratios reaching a final nucleic acid concentration of 650 $\mathrm{nM}$. For obtaining concentrations suitable for in vitro studies, the standard complexes $(650 \mathrm{nM})$ were diluted using low glucose DMEM.

\section{Cell viability and cytotoxicity studies}

3T3-L1 cells were seeded in 96-well plates with a seeding density of $8 \times 10^{3}$ cells. Transfection was performed $24 \mathrm{~h}$ after seeding, by diluting the standard samples $(650 \mathrm{nM})$ to final concentrations of $5,10,25,50,80,100$ and $160 \mathrm{nM}$, followed by incubation intervals of 4 and $8 \mathrm{~h}$. LDH leakage from the cells was measured using the Promega

\footnotetext{
*ivana.ruseska@uni-graz.at
} 
CytoToxONETM assay (Promega, USA). LDH release from cells treated with $9 \%$ Triton X-100 in water was defined as $100 \%$ leakage. For the MTS assay, cells were treated similarly as they were for the LDH assay. Formazan production $4 \mathrm{~h}$ post transfection was measured using the CellTiter96®AQueous One Solution Cell Proliferation Assay (Promega, USA). Formazan production by untreated cells was defined as $100 \%$ cell viability.

\section{Fluorescence imaging}

3T3-L1 cells were seeded on glass bottom dishes and treated with the N-TER peptide alone, or complexed with Fluo-NTC (50 and $100 \mathrm{nM}$ ) for $4 \mathrm{~h}$. Untreated cells served as control. Cells were then fixed with $3.7 \%$ formaldehyde and permeabilized with $0.1 \%$ Triton X-100. Alexa Fluor 488 Phalloidin was used to stain the actin cytoskeleton, and the nucleus was labeled with Hoechst. Images were obtained using CLSM (510 Meta, Carl Zeiss $\mathrm{GmbH})$.

\section{Cellular uptake studies}

3T3-L1 cells were seeded in 96-well plates and transfected using sample concentrations of 5, 10, 25, 50, 80, 100 and $160 \mathrm{nM}$, followed by an incubation period of $2 \mathrm{~h}$, at $4{ }^{\circ} \mathrm{C}$ and $37{ }^{\circ} \mathrm{C}$, with or without ATP-depleting agents (sodium azide and 2-deoxy-Dglucose). After $2 \mathrm{~h}$, the cells were washed once using $100 \mu \mathrm{L}$ PBS and then $100 \mu \mathrm{L}$ of medium was added. Then, all the wells were lysed by $50 \mu \mathrm{L} 0,5 \%$ Triton X-100 in $0.2 \mathrm{M} \mathrm{NaOH}$. The cellular uptake was quantified by measuring the fluorescence intensity using a microplate reader.

\section{Results and discussion}

The aim of our study was to investigate the interactions that take place between the N-TER peptide, used as a delivery system for miRNA, and the cellular membrane, as well as to get some initial insight into its uptake. Using the self-assembly method, complexes between the N-TER peptide and the nucleic acids are formed due to electrostatic interactions. The resulting complexes have a size of 200-300 nm and are positively charged (SchachnerNedherer et al., 2019).
The results obtained from the viability and cytotoxicity studies clearly indicate that the N-TER peptide has no membranolytic activity and the cells' viability seems to be intact in all tested conditions. The previously described antiadipogenic effect of the "naked" peptide, which was assumed to be due to membrane perturbation or mechanical stress (Schachner-Nedherer et al., 2019), is most likely due to the peptide's intrinsic ability to induce cytoskeletal rearrangement. In this way, the peptide increases the membrane fluidity and improves its own cellular uptake (Gerbal-Chaolin et al., 2007). This is in accordance with the images obtained using CLSM, where there is a significant difference in the cytoskeleton of cells treated either with the peptide alone, or complexed with Fluo-NTC, compared to the untreated cells. Furthermore, the conducted uptake studies show a small difference between the uptake at different temperatures, as well as after using ATP-depleting agents, which might indicate that the N-TER peptide leans toward direct translocation as a mode of entry into the cells, which is reasonable after the aforementioned increase in the cellular permeability.

\section{Conclusion}

In vitro studies using the 3T3-L1 cells are performed in order to investigate the interactions and the uptake of the N-TER peptide, as a delivery system for miRNA. The studies have shown that the peptide is safe and suitable as a delivery system. It has an intrinsic ability to influence the cytoskeletal network, increase the membrane's permeability and thus, improve its own cellular uptake, most likely via direct translocation.

\section{References}

Gerbal-Chaolin, S., Gondeau, C., Alrdian-Herrada, G., Heitz, F., Gauthier-Rouviere, C., Divita, G., 2007. First step of the cell-penetrating peptide mechanism involves Rac1 GTPase-dependent actin-network remodeling. Biol. Cell 99, 223-238.

Schachner-Nedherer, A-L., Werzer, O., Kornmueller, K., Prassl, R., Zimmer, A., 2019. Biological activity of miRNA-27a using peptide-based drug delivery systems. Int. J. Nanomed. 14, 7795-7808.

Maced. Pharm. Bull. 66 (Suppl 1) 123 - 124 (2020) 